\title{
Preface: recent developments in financial modelling and risk management
}

\author{
Roy Cerqueti ${ }^{1,2} \cdot$ Rita Laura D'Ecclesia $^{1} \cdot$ Susanna Levantesi ${ }^{1}$
}

Accepted: 18 January 2021 / Published online: 17 March 2021

(c) The Author(s) 2021

In the last decade, a wide range of innovative financial instruments has taken by storm the financial markets. In 2015 for instance, the European Commission (EC) introduced the definition of "innovative financial instruments" as instruments that are complementary to grants or subsidies and as part of a move towards a smarter "funding mix". Loans, equity and quasi-equity instrument and guarantees are considered as a particularly effective way to increase and enhance the impact of EU funding while compared to the traditional grantbased system (EC, 2015), therefore, they represent a way to further promote a more responsible, result-oriented use of European funds by the corporate world.

Cryptocurrencies can actually be considered electronic funding while virtual payment services are showing an impressive growth pattern. Such phenomenon is certainly due to a rapidly growing public interest that creates strong market demand and limits supply. Thousands of developers and dozens of companies are widely involved both in the creation and the use of BlockChain technology. Banks have invested widely in this technology. Research firms expect the user base to grow at an average annual rate of $17.5 \%$ over the next decade, with up to 28.1 billion IoT installed devices by 2020, and revenues exceeding 7 trillion USD the same year (2020). The "new paradigm" for payment systems will bring people to rent a house in just a few minutes: sign the renting contract, perform the payment and get the (digital) keys on their mobile phone.

The willingness and the necessity to implement a more responsible financial market and/or a sustainable economy have strongly affected the dynamics of commodity markets in the last decade. Energy and commodities markets as a whole have represented a key investment tool.

The potential of innovative instruments and the impact of responsible standards ought to be fully exploited while their influence on the markets must be properly and precisely assessed. In addition, an update of portfolio selections methods, an adequate tool for risk measurement and a selection of efficient risk management strategies are all top priorities. Most scholars in the field have been studying and modelling the dynamics of new

Rita Laura D'Ecclesia

rita.decclesia@uniroma1.it

Sapienza University of Rome, Rome, Italy

2 London South Bank University, London, UK 
investment and financing instruments and have provided interesting contributions in the area of financial modelling.

This special issue gathers a selection of refereed papers submitted by the participants to the Stream of Commodities and Financial Modelling at the 29th Euro Conference, held in Valencia, Spain, on July 8-11, 2018. Many scholars, from different countries and with diverse experiences, attended the conference and contributed new insights on some of the most recent developments in Financial Modelling and Risk Management. We received 89 contributions of which 55 have been accepted after two rounds of peer reviews. We have grouped them in five different thematic areas: (1) portfolio selection; (2) pricing; (3) banking; (4) insurance risk management; (5) risk management in commodities and financial markets.

The first section, portfolio selection, integrates twelve papers.

Beraldi, Violi, Ferrara, Ciancio and Pansera address a portfolio optimization problem with rebalancing decisions in response to new market conditions, including transaction costs. Lassance and Vrins propose a higher-moment criterion for portfolio selection based on the exponential Renyi entropy. Corsaro, De Simone and Marino deal with a multiperiod portfolio selection problem by presenting a model based on a fused Lasso approach and introducing two norm penalty terms into the objective function to reduce the costs of the investment strategy. Clemente, Grassi and Hitaj use the network topology to catch the interconnectedness of each asset through the clustering coefficient, thus extending the classical Markowitz mean-variance approach by considering different dependency networks. Naccarato, Pierini and Ferraro consider a pair trading cointegrated strategy exploiting the information from the cointegration between the two price series. Colaneri, Herzel and Nicolosi analyze the expected utility maximization in continuous time and examine how the access to more accurate information regarding market price of risk affects the optimal strategy and estimates what the investor would be willing to pay to receive such information. The importance of the information level owned by investors in determining the market price of risk is also discussed by Altay, Colaneri and Eksi within a dynamic portfolio optimization problem for an investor with a logarithmic utility function.

The increasing availability of big data allows a more efficient asset allocation as suggested by Maggi and Uberti who explored the relationships between web searches (Google search) and some specific social choices that allow the assessment of the role of bad news and measures the risk impact. Flori, Lillo, Pammolli and Spelta deal with a complex network model that is able to describe the behavior of funds' managers and the trading frequency. Barucci, Marazzina and Mastrogiacomo consider a minimum guarantee as a constraint for a fund performance and deal with an optimal investment procedure. Kopa and Rusý offer an asset-liability management model based on a stochastic programming approach, in which a randomness of decision-dependent nature is also taken into account. Vitali and Moriggia set up an Asset/Liability Management model compliant with longterm sustainability using a multi-stage stochastic programming problem.

Section two includes nine papers that provide new insights on pricing.

Few papers try to investigate the pricing features in a behavioral finance framework. Thomann introduces a methodology for improving the performance of trend-following trading strategies. Archibald and Possani focus on the analysis of the actors involved in the investments, by presenting a non-zero sum game between entrepreneurs and investors. Castellano, Mancinelli, Ponsi and Tieri focalize on the neuroscientific aspects of investments, hence pointing to the relevant theme of behavioral finance. In the same behavioral perspective, Kathiravan, Selvam, Venkateswar and Balakrishnan present a study on the effects of the weather variables over the investors' mood, hence on the investment strategies; 
differently, Herteliu, Jianu, Jianu, Bobb, Dhesi, Ceptureanu, Ceptureanu and Ausloos discuss the role played by religiosity on how people perceive the importance of money.

In the context of derivative pricing, Antonelli, Ramponi and Scarlatti deal with the pricing of European options subject to counter-party credit risk, quantifying the credit event in the presence of wrong-way risk. Morelli studies the influence of the multi-curve approach on the interest rate derivative pricing.

Understanding the pricing features of cryptocurrencies is the aim of two papers: Giudici and Polinesi propose a model for the crypto-asset price dynamics based on the correlation matrix between the observed returns that is filtered from noise and employed to derive a clustering structure among prices; Cretarola and Figà-Talamanca model Bitcoin price dynamics in continuous time using a market attention factor that is proven to boost in a bubble under suitable conditions.

Section three includes eight papers that address the recent debate on banking performance and the role of the ongoing updating of the regulatory framework.

Flori, Giansante, Girardone and Pammolli discuss how the classification of banks in terms of their business provides banks' distresses forecast. du Jardin deals with a bankruptcy forecasting model for firms obtained through a biclustering method. Interestingly, the author discusses the existing models for bankruptcy prediction on the basis of the different natures of bankruptcy. Girardi and Ventura use an econometric model to understand the credit crunch in Italy and analyze the economic and financial implications on institutions and regulations. The analysis of the interconnections with the economic content is studied by Cinelli, Ficcadenti and Riccioni; the role of risk sharing-based reform of the Eurozone-with a new role for the European Stability Mechanism-is suggested by Dosi, Minenna, Roventini and Violi. Yan, Zhang and Wang study the allocation problem for banks under regulations on uncertain migration risk of credit ratings and capital adequacy ratios, while Morelli analyses the role of liquidity in the framework of the Basel III regulation. The network of interbank exposures is the focus of Cinelli, Ferraro, Iovanella and Rotundo who deal with the issue of robustness against failure cascades. They formulate a model of financial contagion in a framework of incomplete information to estimate the width and length of the cascades.

Section four addresses the issue of risk management in the insurance business taking into account for the changing framework. Four papers provide interesting insights in this area.

The capital allocation model under Solvency II Directive 2009/138/EC is proposed in Baione, DeAngelis and Granito while Devolder, Levantesi and Menzietti deal with the exploration of the automatic balance mechanisms for the Notional Defined Contribution. D’Amato, Di Lorenzo, Haberman, Sibillo and Tizzano conceptualize a contract based on real estate rights for having a life annuity in exchange of a single premium. Altuntas, Berry-Stölzle and Cummins discuss the implications of the employment of the enterprise risk management approach in the German property-liability insurance companies.

Section five, risk management in commodities and financial markets, integrates twentytwo papers of which six deal with energy risk management.

Benedetti, Biffis, Chatzimichalakis, Fedele and Simm capture the potential impact of carbon pricing on fossil fuel stocks, then used to create Smart Carbon Portfolios according to Bayesian and Black-Litterman methodologies. Lucheroni and Mari propose a scheme for hedging and managing production costs of a risky electricity generation portfolio, initially assumed to be dispatchable, to which intermittent electricity generation from non-dispatchable renewable sources is further added. Cerqueti and Fanelli discuss the usefulness of the 
long-term memory properties in price prediction of commodities portfolios. Maier evaluates natural resource investments under uncertainty by re-visiting the well-known example of a copper mine project under different commodity price models using the influence diagram simulation-and-regression approach. Franzoni and Pelizzari use weather derivatives to manage rainfall risk of the hospitality industry. They propose an innovative pricing model for such instruments. Jotanovic and D'Ecclesia study the European natural gas market and its recent developments showing how it is converging toward a single market as requested by the European Community.

Shanker and Satir concentrate on the management of foreign exchange risk modelling, the optimal choice of the terms of a risk-sharing supply contract between the buyer and the supplier of a product, who are located in different countries. Alexander and Chen address the model risk inherent in the assumptions that are made for the decision in real option valuation. Lai, Chen and Sun using comonotonicity tests show that expected stock returns do not increase monotonically with lower volatility, but volatility increases with specific risks. D'Ecclesia and Clementi propose an Artificial Neural Network approach to track stock returns implied volatility showing how it provides a more efficient tool in terms of tracking error. Browne, Hofmann, Richter, Roth and Steinorth analyze peer effects in risk preferences using data from the German Socio-Economic Panel, shedding light on the determinants of economic preferences.

Within a complex networks setting, Cerqueti, Clemente and Grassi propose a novel measure of systemic risk that is defined according to the structure of clustered neighbors around the nodes of the network, thus incorporating the generalized concept of high order clustering coefficient.

Ceptureanu, Ceptureanu and Herteliu explore the role of networking with stakeholders in facilitating medium-sized enterprises' access to external financing and international markets.

Under a purely statistical-econometric point of view, financial time series show peculiarities, regularities and relevant properties. Mamatzakis and Tsionas introduce Bayesian inference procedures based on a Sequential Monte Carlo approach for observing financial stability. Benedetto, Mastroeni and Vellucci deal with the connection between the information content of two different financial time series by the introduction of two new entropic metrics. Fallahgoul and Loeper compare the performance of some parametric models driven by stable and tempered stable distributions and investigate the behaviour of assets returns on the tails of their distribution. Norton, Khokhlov and Uryasev present formulas for the buffered probability of exceedance and the superquantile for a large set of univariate probability distributions and apply their outcomes to density estimation and portfolio optimization. Guidolin and Pedio implement a stepwise regression procedure for assessing whether the predictive power for commodity futures can be improved by using commodity-specific variables instead of macroeconomic ones. A new suggestion to cluster financial series is proposed by Cappelli, Di Iorio, Maddaloni and D'Urso, and by D'Urso, De Giovanni and Massari. The first presents a procedure based on Atheoretical Regression Trees for identifying groups of firms on the basis of their risk profiles, while the latter deals with multivariate financial time series and employ a clustering distance able to neutralize the effects of the outliers.

Dhesi, Shakeel and Ausloos perform forecasting exercises on assets returns by employing a modified version of the Brownian motion that is also able to include an irrationality component. Brigo, Pisani and Rapisarda offer a generalization of the Multi Variate Mixture Dynamics model, by also providing a definition of correlation skewness under the proposed perspective. 
Funding Open access funding provided by Università degli Studi di Roma La Sapienza within the CRUICARE Agreement.

Open Access This article is licensed under a Creative Commons Attribution 4.0 International License, which permits use, sharing, adaptation, distribution and reproduction in any medium or format, as long as you give appropriate credit to the original author(s) and the source, provide a link to the Creative Commons licence, and indicate if changes were made. The images or other third party material in this article are included in the article's Creative Commons licence, unless indicated otherwise in a credit line to the material. If material is not included in the article's Creative Commons licence and your intended use is not permitted by statutory regulation or exceeds the permitted use, you will need to obtain permission directly from the copyright holder. To view a copy of this licence, visit http://creativecommons.org/licenses/by/4.0/.

Publisher's Note Springer Nature remains neutral with regard to jurisdictional claims in published maps and institutional affiliations. 